1 Richardson JR, Ferguson J, Hiscox J, et al. Noninvasive assessment of cardiac output in children. $\mathcal{F}$ Accid Emerg Med 1998;15:304-7.

2 Childs C, Goldring S, Tann W, et al. Suprasternal Doppler ultrasound for the assessment of stroke distance. Arch Dis Child 1998;79:251-5.

3 Little RA, Kirkman E, Driscoll P, et al. Preventable deaths after injury: why are the traditional "vital" signs poor indicators of blood loss? $f$ Accid Emerg Med 1995;12:1-14.

4 Mowat DHR, Haites NE, Rawles JM Aortic Mowat DHR, Haites NE, Rawles JM. Aortic blood velocity measurement in healthy adult Res 1983;17:75-80.

\section{Safe use of thrombolysis in acute} myocardial infarction

EDITOR,-The safe administration of thrombolysis in acute myocardial infarction (AMI) is a difficult area, and the contribution by Wald is not helpful. ${ }^{1}$ The author criticises the lack of evidence based practice among junior doctors in an area in which generally accepted evidence based guidelines are lacking. The author concludes that junior doctors probably under-utilise thrombolysis, but presents no evidence to support this; the responses to this kind of questionnaire survey do not constitute evidence of current practice.

We agree that practice should be evidence based whenever possible; many of the perceived contraindications to thrombolysis are not evidence based, and may be said to err on the side of caution. However, it is important to appreciate that thrombolysis trials use a selected group of patients, and a lack of evidence of harm in a selected group does not constitute evidence of harmlessness when applied to the general population.

In Sheffield, thrombolysis in AMI has been audited in detail. We withhold thrombolysis within two months of a cerebrovascular accident, after recent head injury, and in uncontrolled hypertension, cerebral aneurysm, or tumour. In spite of this our cerebrovascular accident rate is $2.3 \%$, higher than in the major trials. The risk of a cerebrovascular accident after thrombolysis is not an "intuitive fear", it is a real risk.

We have a written protocol and contraindications checklist to guide our junior doctors, with contraindications broadly similar to those presented by Wald. ${ }^{1}$ In the last year, only $2.5 \%$ of patients admitted with AMI had treatment withheld because of a contraindication. However, this group had a 30 day mortality of $45 \%$ (compared with $15.8 \%$ in thrombolysed patients).

The decision to withhold thrombolysis should be not made solely by a junior doctor. When the risk from thrombolysis is judged to be significant, urgent discussion with cardiologist is appropriate. Systemic throm bolysis is not the only method of achieving reperfusion. The interests of these patients may be best served by exploring the obstacles preventing immediate angiography.

J EDHOUSE

Senior Registrar, Accident and Emergency Medicine, Accident and Emergency Department, Northern General Hospital, Herries Road, Sheffield $S 57 A U$

1 Wald DS. Perceived contraindications to thrombolytic treatment in acute myocardial infarction. A survey in a teaching hospital. 7 Accid Emer Med 1998;15:329-31.

The author replies

The survey shows a clear difference in opinion, among junior medical doctors, as to what constitutes a reason to witholdthrombolysis in AMI. This uncer- tainty reflects a lack of evidence on the harmful effects of thrombolytic therapy. Where there are risks, but the benefits of treatment are known to outweigh them, treatment should be given rather than withheld. This should be the message delivered to doctors responsible for managing patients with AMI.

I agree with Miss Edhouse that junior doctors should not withhold thrombolysis in AM without discussion with a senior colleague, and that primary angioplasty and intracoronary stenting may provide alternative strategies for patients in whom thrombolysis is withheld.

Capnography and "major" accident and emergency departments in East Anglia

EDIToR,-This year, Advanced Trauma Life Support (ATLS) courses based on the sixth (1997) edition of the ATLS manual ${ }^{1}$ have been held. One major change from the previous edition is the recommendation for capnography (end tidal carbon dioxide monitoring) in intubated patients. Capnography is the "gold standard" for the correct siting of the endotracheal tube in the airway. Such devices have been used in anaesthetic departments for some years, and are recommended by the Association of Anaesthetists in thei guidelines for safe practice. ${ }^{2}$ Its importance has been emphasised recently in a case before the General Medical Council. The absence of end tidal carbon dioxide monitoring was cited as evidence in support of a claim of negligence against an anaesthetist involved in a perioperative death.

In a telephone survey of 10 "major" accident and emergency (A\&E) departments in East Anglia (as described by the British Association of Accident and Emergency Medicine ${ }^{4}$ ) only five of the 10 units had capnography available, despite all departments $(100 \%)$ employing at least one ATLS provider at staff grade, registrar, or senior house officer level. In seven of the departments, the $A \& E$ consultant was known to be an ATLS course instructor. Six of the 10 hospitals offered an ATLS course in the last year (data from telephone survey and personal communication from the Royal College of Surgeons of England), and six described immediate access to a multidisciplinary "trauma team" (typically with anaesthetic surgical, and orthopaedic middle grade doctors), with the remaining four able to enlis immediate help from the duty anaesthetist via a pager system

It appears that since only $50 \%$ of major A\&E departments in East Anglia have cap nography available, half of the departments do not have the recommended equipment to allow ATLS providers and anaesthetists to provide the latest advanced airway care.

It is understood, however, that at least some of the departments contacted are currently considering the acquisition of a capnograph.

ROBERT FLORANCE

Specialist Registrar, Accident and Emergency RICHARD GRIFFITHS

Consultant Anaesthetist

ANDREW COPE

Consultant, Accident and Emergency Peterborough District Hospital Thorpe Road,

Peterborough PE3 6DA 1 American College of Surgeons. Advanced trauma can College of Surgeons, 1997.
2 Association of Anaesthetists of Great Britain and Ireland. Recommendations for standards of monitoring during anaesthesia and recovery. Association of Anaesthetists of Great Britain and Ireland, 1994.

3 Anonymous. Doctor guilty after girl, 10, dies at dentist. Independent 13 June 1998.

4 British Association for Accident and Emergency Medicine. Directory 1996. London: British Association for Accident and Emergency Medicine, 1996.

Violence in the accident and emergency department: liaison with the police

EDITOR,-In common with colleagues throughout the country, staff in the accident and emergency (A\&E) department at Leighton Hospital have become increasingly concerned with the rising levels of violence and verbal abuse and have considered various security measures, including the use of closed circuit television and security staff.

Security staff are extremely expensive and their powers are very restricted.

Relationships with the local police have always been good and they have been aware of the increasing problems we face. Continuing discussion has resulted in a police base, which was previously sited some two miles from the hospital, being relocated in the grounds of the hospital, immediately adjacent to the $A \& E$ department. Consequently, police are immediately available for 126 hours per week (8.00 am to 2.00 am, seven days per week).

This arrangement ensures an immediate police presence in the $\mathrm{A} \& \mathrm{E}$ department for $75 \%$ of the time. The hospital has agreed to provide accommodation (a Portakabin), costing $£ 5000$ per annum, and car parking facilities. The other expenses are borne by the police, who are obviously able to continue with their normal duties.

Additional benefits of this arrangement include improved relationships with the police (including the coroner's officer) and the deterrent effect of the obvious police presence.

We believe that this arrangement is unique and provides a superb level of security for minimal financial cost.

JOHN BACHE

Consultant in Accident and Emergency Medicine, Accident and Emergency Department, Leighton Hospital, Crewe, Cheshire CW1 4Qf

DUNCAN CHAMBERS

Consultant in Accident and Emergency Medicine PETER GUILDFORD

Chief Inspector (Operations Manager, Crewe Division), Cheshire Constabulary

BOOK REVIEWS

The Handbook of Medical Care of Catastrophes. By J S P Lumley, J M Ryan, P J Baxter, and N Kirby. (Pp 223; $£ 20.00$.) Royal Society of Medicine, 1996. ISBN 1 8535296 X.

There is a large void between the thought that one could be of value at a catastrophe, and the challenge to be more of a help than a hindrance. 